\title{
Fluorescence spectroscopic detection and measurement of single telomere molecules
}

\author{
Cyrus W. Beh ${ }^{1, \dagger}$, Ye Zhang ${ }^{1, \dagger}$, Yun-Ling Zheng' ${ }^{2}$, Bing Sun ${ }^{2, *}$ and Tza-Huei Wang ${ }^{1,3,4, *}$ \\ ${ }^{1}$ Department of Biomedical Engineering, Johns Hopkins University, Baltimore, MD 21218, USA, ${ }^{2}$ Department of \\ Oncology, Lombardi Comprehensive Cancer Center, Georgetown University Medical Center, Washington, DC 20057, \\ USA, ${ }^{3}$ Department of Mechanical Engineering, Johns Hopkins University, Baltimore, MD 21218, USA and ${ }^{4}$ Institute \\ for NanoBioTechnology, Johns Hopkins University, Baltimore, MD 21218, USA
}

Received December 29, 2017; Revised June 18, 2018; Editorial Decision June 28, 2018; Accepted June 28, 2018

\begin{abstract}
Telomeres are the end-caps of chromosomes that serve to protect the integrity of the genome. Below certain critical lengths, the telomeres can no longer fulfill their protective function, and chromosomal instability ensues. Telomeres shorten during normal cell division due to the end replication problem and are implicated in the development of various aging-associated diseases, including cancer. Telomere length has the potential to serve as a useful biomarker in the field of aging and cancer. However, existing methods of telomere measurement are either too laborious, unable to provide absolute measurement of individual telomere lengths, or limited to certain chromosomes or cell types. Here, we describe an easy single-molecule, fluorescence spectroscopic method for measuring the length of telomeres that permits the profiling of absolute telomere lengths in any DNA sample. We have demonstrated the accurate detection of telomeres as short as 100 bp using cloned telomere standards, and have profiled telomere lengths in human cancer cell lines and primary cells. Since this method allows direct comparison between samples, it could greatly improve the clinical utility of telomere biomarkers.
\end{abstract}

\section{INTRODUCTION}

Telomeres are specialized DNA tandem repeats and protein structures that cap the chromosomal ends, and protect the chromosomes from degradation by serving as sacrificial bases during each cell replication. The rate of attrition of these tandem repeats is $\sim 50-200 \mathrm{bp}$ with every cell division (1). When telomeres become critically short, they trigger DNA checkpoint responses mediated by telomere- associated proteins that prevent further cell replication (Figure 1A(i)) (2-4).

Dysfunction of telomeres can take the form of premature shortening (as in many hereditary telomere syndromes such as Dyskeratosis Congenita, Hoyeraal-Hreidarsson syndrome and pulmonary fibrosis) (2,3) or lengthening. The latter frequently occurs in cancer, and is preceded by failure to arrest replication in the presence of critically short telomeres $(5,6)$, and the rescue of ensuing cellular crisis by activation of either telomerase (85-90\% of tumors) or proteins associated with alternative lengthening of telomeres (ALT, $10-15 \%$ of tumors) $(4,6,7)$. This in turn permits the cells to multiply without constraint.

Telomere length (TL) is also of great interest in the context of the ageing process. However, results from studies using TL to test a host of hypotheses related to the biology of human ageing have often been inconsistent. In using average TL as the only parameter, these studies fail to take into account the heterogeneity of TLs on chromosome arms, first reported by Lansdorp et al. (8). In fact, it is increasingly recognized that the deleterious effects of telomere dysfunction are mediated by the load of critically short telomeres (9-14). For example, genetic studies in mice have shown that the shortest telomeres, rather than the average TL, are critical for chromosome stability and cell viability, and are likely a major cause of age-related pathologies (10). This load can increase due to the gradual shortening of telomeres across all chromosomal arms during normal cellular aging. However, even without significant differences in average TL, the load of critically short telomeres between samples can differ significantly due to variations in the shape of the TL distribution, either between individuals or cell types (Figure 1A(ii)), or because of bi- or multi-modal distribution that may occur as a result of catastrophic telomere loss (15-17), or species-specific telomere biology (Figure 1A(iii)) (18). Therefore, determining the distribution of absolute TLs is of great importance.

\footnotetext{
${ }^{*}$ To whom correspondence should be addressed. Tel: +1 410516 7086; Fax: +1 410516 7254; Email: thwang@jhu.edu

Correspondence may also be addressed to Bing Sun. Email: bs464@georgetown.edu

${ }^{\dagger}$ The authors wish it to be known that, in their opinion, the first two authors should be regarded as joint First Authors.
} 
A

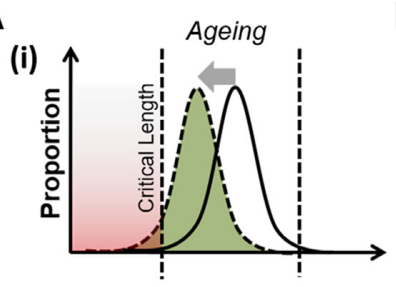

(ii)

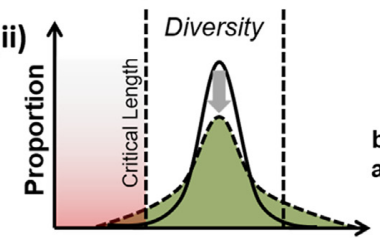

(iii)

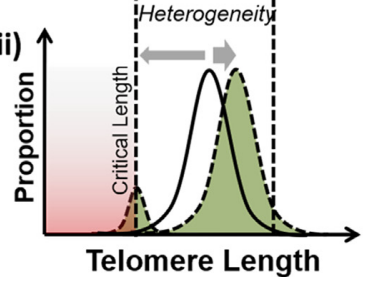

B Genomic DNA XWOWM Telomere $N M W M N D$ Biotin Probe<smiles>CC1CC2CCC1C2</smiles>

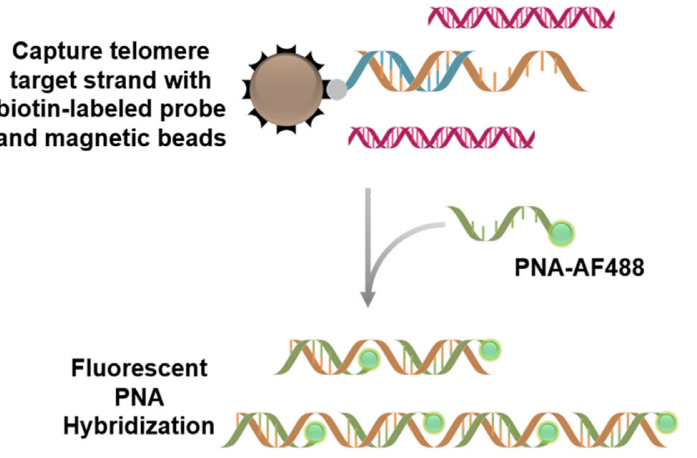

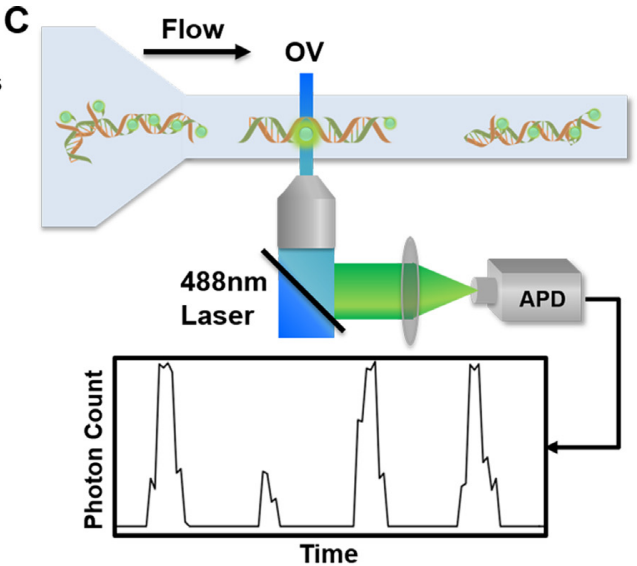

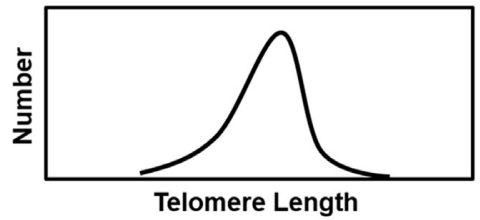

Figure 1. Principle of telomere measurement by PHAST. (A) The biological effects of telomeres are mediated primarily by the proportion of telomeres below a critical length. (i) This most commonly happens in ageing, where the telomeres in a cell population shorten with doubling. However, the proportion of short telomeres can also reach critical levels when the average telomere length is normal. (ii) This can happen due to the naturally occurring diversity in the shape of the distribution between individuals, or (iii) if the telomere distribution is highly heterogeneous. (B) To perform our assay, cells are lyzed to release the DNA from the nucleus, and incubated with biotinylated PNA probes. Telomeric DNA is separated from genomic DNA using magnetic beads, and released after washing, whereupon fluorescent probes are hybridized to the telomeric sequences. (C) The labelled DNA is then flowed through a microchannel, and excited by a laser through an objective as it transits the observation volume (OV). These peaks can then be processed to yield the telomere distribution.

Apart from monitoring natural TL changes, the prospect of telomere-lengthening treatment for various medical conditions and prolonging longevity has been raised in recent years. This can be achieved by transfecting cells with telomerase reverse transcriptase (TERT), resulting in significant telomere lengthening $(19,20)$. In mouse studies, introducing TERT in adult and old mice resulted in improvements in all aging markers, increased longevity, without any increased cancer incidence (20). A more recent study also raised the possibility of reducing telomere attrition, or even elongating telomeres in patients with various telomere diseases using androgen therapy (21). While questions of safety (including the risks of cell immortalization) and efficacy (how and when to introduce the treatment) remain to be answered, these applications argue for the need for a method for determining TL profiles that has a higher throughput.

The strengths and weaknesses of existing TL measurement methods, which have been well documented in literature, are briefly summarized below $(13,22)$. Despite their high sensitivity, quantitative polymerase chain reaction (qPCR) methods using primers specific to the telomere repeat sequence yield only average TL for a given sample $(23,24)$. Flow-fluorescence in situ hybridization (FlowFISH) fluorescently labels telomeres in blood cells that are then analyzed by flow cytometry, and gives the distribution of total TL in each cell $(22,25,26)$. Neither method can detect small fractions of critically short telomeres among all chromosomal ends, arguably the most important telomere biomarker. Although terminal restriction fragments (TRF)/Southern blot analysis can in principle yield infor- mation of the size distribution of telomeres, it is only semiquantitative, with relatively poor sensitivity, particularly for shorter telomeres $(27,28)$. Furthermore, the size estimates are skewed by the presence of a sub-telomeric sequence of variable size on each fragment, limiting their utility (Supplementary Notes 6 and 8) (28).

A newer PCR-based approach known as single telomere length analysis (STELA) using chromosome-specific primers provides the TL of specific chromosomal arms $(22,29)$. However, STELA fails to adequately capture the complete picture of the TL dynamics within cells since primers exist for less than one-fifth of all chromosomal arms (22). Lastly, quantitative FISH (q-FISH) is able to provide an abundance of information, including chromosomal armspecific TL. However, the method requires cells that can be induced into metaphase, and thus precludes its application to archival samples such as frozen DNA samples. Furthermore, sample preparation is very time consuming and labor intensive, thus severely limiting its practical applications (22).

Against this technical backdrop we have developed a method dubbed peptide nucleic acid (PNA) Hybridization and Analysis of Single Telomere (PHĀST) assay to determine the length of telomeres, hybridized with fluorescent probes, via analysis of single telomere fluorescence measured by a light-sheet fluorescence spectroscopic method $(30,31)$. Starting with genomic DNA, the entire sample preparation procedure takes less than 3 hours, including a 2-h PNA hybridization interval. Telomere molecules are detected one at a time as they transit an observation vol- 
ume $(O V)$, generating single-molecule fluorescence bursts. The properties of these bursts are then analyzed and used to gate the telomere populations, in an approach akin to flow cytometry, to remove spurious bursts and improve the detection limit. By leveraging the unique capabilities of single molecule detection, we have been able to determine the telomere size distribution with DNA from small numbers of cells.

\section{MATERIALS AND METHODS}

\section{CICS instrumentation}

The instrumentation for CICS is identical to that previously reported (30,31). Briefly, an Ar-ion laser (488 nm, Melles Griot, Carlsbad, CA, USA) is passed through an optical fiber and additional shaping optics that expand the laser spots into large illumination discs, which are then focused into a light sheet using a cylindrical lens. The power output as the beam exits the coupler is adjusted to $2.5 \mathrm{~mW}$ prior to each experiment. The light is then focused into a microfluidic channel using a $100 \times$ oil immersion microscope objective (UPlanFl, Olympus, Center Valley, PA, USA). The objective collects the emitted photons from the sample in an epifluorescence configuration, and a dichroic mirror (z488/633rpc, Chroma Technology, Bellows Falls, VT, USA) is used to separate the excitation light and emission fluorescence. A confocal aperture $(600 \times 150 \mu \mathrm{m}$, National Aperture, Salem, NH, USA) is used to spatially filter out out-of-plane light, yielding an effective detection volume of $7 \times 2 \mu \mathrm{m}$ on the microfluidic chip. Finally, dichroic mirrors and band-pass filters (Omega Optical, Brattleboro, VT, USA) are used to spectrally separate the green and red fluorescence and to select the desired wavelengths for detection on the avalanche photodiode (APD SPCM-AQR13, Perkin Elmer, Vaudreuil, Quebec, Canada). Fluorescence data is collected and processed using custom software using the Labview interface.

\section{Telomeric standards generation}

Telomere length standards were synthesized through a repeated extension process. Initial telomere template (90 bp) was synthesized by Integrated DNA Technologies, Inc. (Coralville, IW, USA), with KpnI and XhoI site at $5^{\prime}$ end and SalI at $3^{\prime}$ end containing 12 telomere repeats (GGTA CCTCGAGGG-(TTAGGG) 12 -TCGAC). Two additional forward (5'-TGGTACCTCGAGGGTTAGG-3') and reverse $\left(5^{\prime}\right.$-GTCGACCCTAACCCTAACC- $\left.3^{\prime}\right)$ primers that can anneal to the telomere template at both ends were synthesized and used in a PCR reaction to create a double strand telomere template which was then ligated into pCR4-TOPO TA vector (Life Technologies, Grand Island, NY, USA), and sequence verified by Sanger DNA sequencing (Genwiz, Germantown, MD, USA). The telomere template insert was then released from the TA vector by XhoI and SalI digestion. After separation by electrophoresis on a $1.5 \%$ agarose gel and band purified with a DNA gel purification kit from Qiagen, the double-strand template was further cloned into the XhoI site of pUC19 (New England Biolabs, Ipswich, MA (NEB)). To use as a subsequent hosting vector, this obtained plasmid was cut with XhoI.
To prepare telomere insert for extension, the cloned insert in the plasmid was released with KpnI and SalI, or by amplification with PCR using the plasmid as template and two primers annealing to pUC19 beside the cloned insert (pUC19-F: 5'-AGTGAATTCGAGCTCGGTAC-3' and pUC19-R: 5'-CAAGCTTGCATGCCTGCAG-3') followed by digestion with SalI. Finally, the prepared hosting vector and telomere extension insert were mixed and fused together with a Gibson Assembly cloning kit (NEB) to have the telomere insert in the hosting vector extended. This process was repeated several times in order to obtain longer telomere inserts. To check the length, telomere inserts were released with XhoI and SalI digestion and run on a $1.5 \%$ agarose gel.

\section{DNA digestion by restriction enzyme}

To digest the DNA samples, we incubate $20 \mu 1$ of the DNA (typically between 1 and $5 \mu \mathrm{g}$ total DNA) with $4.39 \mu \mathrm{l}$ of a mixture of RsaI and HinfI restriction enzymes (Roche Applied Sciences, 1000 units) and Buffer A (Roche) (1:1:2 volume ratio) at $37^{\circ} \mathrm{C}$ for $2 \mathrm{~h}$.

\section{Single-tube purification and hybridization}

To minimize the DNA fragmentation during telomere preparation, we chose to apply phenol/chloroform extraction, which is considered as the gold standard to produce high-purity and high-molecular weight DNA with minimal shearing. Genomic DNA is extracted from cell lines using phenol/chloroform extraction and added to a 150 $\mu 1$ proprietary PNA hybridization buffer containing 500 pM of telomere-specific biotinylated PNA probes (pcTelBio, PNA Bio Inc.). After a $10 \mathrm{~min}$ incubation at $85^{\circ} \mathrm{C}$, the mixture is left at room temperature for $30 \mathrm{~min} .20 \mu \mathrm{l}$ of streptavidin-coated magnetic Dynabeads C1 (Life Technologies) is then washed in $1 \times$ binding washing (BW) buffer as described by manufacturer, resuspended in $150 \mu 12 \times \mathrm{BW}$ buffer and incubated with the DNA/PNA mixture for 30 min on a rotating sample holder. The pulled-down DNA is then washed with $1 \times \mathrm{BW}$ buffer to isolate the telomerecontaining DNA.

\section{Fluorescent PNA hybridization}

DNA samples in solution are mixed directly with $500 \mathrm{nM}$ Alexa 488 labeled PNA (PNA-AF488, PNA Bio Inc.) in PNA hybridization buffer, incubated at $85^{\circ} \mathrm{C}$ for $10 \mathrm{~min}$ and left at room temperature in the dark for $2 \mathrm{~h}$ to complete the labeling. For DNA samples bound to magnetic beads, the telomeric DNA is released from the pcTel-Bio/Dynabeads upon heating, and placed on a magnetic holder prior to incubation at room temperature to isolate the telomeres from the beads. The large excess of PNA-AF488 ensures minimal re-hybridization onto the beads. Finally, the samples are purified with $\mathrm{S} 400-\mathrm{HR}$ microspin columns (GE Healthcare) to remove the excess PNA-AF488.

\section{Telomere sizing on CICS}

Microfluidic devices are fabricated as described previously (30-32). The purified samples are flowed through a $5 \mu \mathrm{m}$ 
$\times 0.5 \mu \mathrm{m}$ microfluidic channel $(\mathrm{w} \times \mathrm{h})$ using a pressure controller (Alicat Scientific) and detected on CICS. Emitted photons are counted at $0.1 \mathrm{~ms}$ intervals. To ensure representative data, the pressure is adjusted to ensure that the average transit time of the samples through the CICS detection volume is at least $3 \mathrm{~ms}$. Gating to exclude spurious peaks is performed as described in Supplementary Note 5. A standard curve is constructed using linearized cloned telomere standards $(0.1,0.2,0.4,0.9,1.2$ and $2.4 \mathrm{~kb}$ TL $)$, relating the height of the fluorescence peak to the length of the DNA. We have found that the fluorescence peak height varied linearly with TL, and is invariant between experiments. Therefore the same standard curve is used for size estimation. For TLs exceeding the range of the standards, a linear extrapolation is used to estimate their sizes as discussed in the Supplementary Note 3 . In addition, $3 \mu \mathrm{g}$ of HeLa genomic DNA (NEB) was processed using our PHAST assay as described above and the resultant telomere length was compared with that measured by TRF (Supplementary Note 8).

\section{Determination of pull-down efficiency}

To determine the efficiency of the pull-down assay, a mixture of telomeric standards $(0.2,0.4,1.2$ and $2.4 \mathrm{~kb})$ at high (1.60, 1.64, 1.84, $2.16 \mathrm{ng}$ respectively) and low concentrations $(40,41,46,54 \mathrm{pg}$ respectively) was mixed with $1 \mu \mathrm{g}$ of heat-fragmented genomic DNA $(\sim 100-1000 \mathrm{bp})$ prior to sizing with PHAST assay (200 $\mu$ l final volume). As controls, telomeric standards was mixed at the same proportions and hybridized with PNA-AF488, without going through the pull-down process. The pull-down efficiency of each species is then determined by comparing the number of molecules detected with and without pull-down.

\section{Comparison of PHAST with q-FISH}

WI38, U2OS and R83 cells were purchased from ATCC (Manassas, VA, USA) and cultured in the medium as recommended by the vendor. Human primary fibroblasts were the gift of Drs Xuefeng Liu and Richard Schlegel in the Molecular Oncology Program, Lombardi Comprehensive Cancer Center, Georgetown University. The cells were cultured in DMEM $+10 \% \mathrm{FBS}$, and harvested at metaphase, followed by chromosome preparation according to the method described previously (33). The same batch of cells in separate flasks were harvested with $0.1 \%$ Trypsin/EDTA (Invitrogen, Rockville, MD, USA), and genomic DNA from cell pellet were purified with DNA purification kit (Qiagen, Germantown, MD, USA). The quality and concentration of the DNA was measured with Nanodrop.

Chromosome arm-specific telomere length was measured by telomere quantitative fluorescence in situ hybridization (q-FISH) as previously described (34). Briefly, chromosome preparations were dropped onto clean microscopic slides and hybridized with $15 \mu$ l of hybridization mixture consisting of $0.3 \mu \mathrm{g} / \mathrm{ml}$ Cy3-labeled telomere-specific PNA probe (PNA Bio Inc.), $1 \mu$ l of cocktails of FITC-labeled centromeric PNA probes specific for chromosomes 2, 4, 8, 9, $13,15,18,20$ and 21 and $20 \mu \mathrm{g} / \mathrm{ml}$ of Cy3-labeled centromeric PNA probes specific for chromosome X (Biomarkers, Rockville, MD), in 50\% formamide, $10 \mathrm{mM}$ Tris- $\mathrm{HCl}$,
$\mathrm{pH} 7.5$, and 5\% blocking reagent. Slides were denatured and then hybridized at $30^{\circ} \mathrm{C}$ for $3 \mathrm{~h}$. After hybridization, the slides were sequentially washed 10 minutes each at $42^{\circ} \mathrm{C}$ : once in $1 \times \mathrm{SSC}$, once in $0.5 \times \mathrm{SSC}$, and once in $0.1 \times$ SSC. The slides were then mounted in anti-fade mounting medium containing $300 \mathrm{ng} / \mathrm{ml}$ DAPI.

After q-FISH, cells were analyzed using an epifluorescence microscope equipped with a charge-coupled device (CCD) camera. Metaphase cells were captured with exposure times of $0.15,0.25$ and $0.05 \mathrm{~s}$ for Cy3, FITC and DAPI signals, respectively. Digitized metaphase images were analyzed using the Isis software (MetaSystems Inc. Boston, MA, USA), which permits the measurement of 92 telomere signals simultaneously after karyotyping. Telomere fluorescence intensity units (TFU) were recorded as an indirect measurement of telomere length. For each sample, at least 12 metaphase cells were analyzed. Each pair of homologous telomeres was recorded separately as homologous short (S) and homologous long (L).

PHAST assay was performed on three cell lines, namely WI-38, R83 and U2OS. A total of around $5 \mu \mathrm{g}$ genomic DNA is processed as previously described. q-FISH results were obtained from our collaborators at Georgetown University. Since q-FISH does not provide absolute telomere length, the TFU are scaled to match the mode of the TFU histogram to the telomere length histogram calculated using PHAST.

Similarly, PHAST assay was performed on three clinical samples (two from cancer patients, one from a control subject). Genomic DNA was isolated from three clinical blood samples (BA01628, BA01650, CB3319) obtained from our collaborators at Georgetown University. For each clinical sample, a total of around $500 \mathrm{ng}$ genomic DNA was processed as described previously. Q-FISH results were also obtained from our collaborators who offered the clinical samples for comparison.

\section{Telomeric sequence generation via rolling circle amplification}

It is difficult to obtain telomere standards longer than $2.4 \mathrm{~kb}$ because the cloning vector is unable to accept a much larger cassette. As a result, we employed padlock probe chemistry (35) and Rolling Circle Amplification (36) to produce longer telomeric sequences. We design our padlock probe to contain two non-telomeric regions at $5^{\prime}$ and $3^{\prime}$ ends, and an internal part consisting of 9 repeats of CCCTAA sequences. In the presence of oligo sequence complementary to the non-telomeric regions, padlock probe is ligated to a closed circle for subsequent amplification and result in single strand DNA over $20 \mathrm{~kb}$, a substantial proportion of which are telomeric sequences. The sequence of our padlock probe is $5^{\prime} /$ Phos/-GGAACCCGATATAATCCGCCC TTCССТАACCCTAACCCTAACCCTAACCCTAA CCCTAACCCTAACCCTAACCCTAAATATTG TGTTGAAACACCGCCC. The sequence of RCA primer is 5'-GGGTTAGGGTTAGGGTTA and the sequence of complementary non-telomeric oligo is 5'-AAGGGCGGATTATATCGGGTTCCGGGCGG TGTTTCAACACAATATGGCGG. All DNA oligonucleotides were purchased from Integrated DNA Technologies, Inc. (Coralville, IA). Reagents for ligation and RCA 
reactions, including $9^{\circ} \mathrm{N}^{\top M}$ DNA Ligase, $10 \times 9^{\circ} \mathrm{N}^{\top M}$ DNA Ligase Reaction Buffer, Phage ø29 DNA Polymerase, Exonuclease I (E. coli), Exonuclease III (E. coli) and 10 $\times$ Isothermal Reaction Buffer were purchased from New England BioLabs, Inc. (Ipswich, MA, USA).

Padlock probe hybridization and ligation was achieved in a $25-\mu 1$ reaction mixture contained $150 \mathrm{nM}$ of padlock probe, $400 \mathrm{nM}$ of complementary non-telomeric oligo, 0.4 Units $/ \mu 19^{\circ} \mathrm{N}^{\top \mathrm{M}}$ DNA Ligase, and $1 \times 9 \mathrm{~N}$ DNA Ligase Reaction Buffer $(10 \mathrm{mM}$ Tris- $\mathrm{HCl}, 600 \mu \mathrm{M}$ ATP, $2.5 \mathrm{mM}$ $\mathrm{MgCl}_{2}, 2.5 \mathrm{mM}$ dithiothreitol, $0.1 \%$ Triton X-100, pH 7.5 at $25^{\circ} \mathrm{C}$ ). Padlock probe hybridization and ligation was performed at $60^{\circ} \mathrm{C}$ for $1 \mathrm{~h}$. Then $25 \mu \mathrm{l}$ of the ligation product was treated with 10 units of Exonuclease I and 50 units of Exonuclease III at $37^{\circ} \mathrm{C}$ for $1 \mathrm{~h}$ to remove extra padlock probes that were not circularized. After digestion, $5 \mu \mathrm{l}$ of the enzyme-treated ligation product was mixed with $200 \mathrm{nM}$ RCA primer, $400 \mu \mathrm{M}$ dNTPs, $10 \mathrm{U} / \mu 1$ Phage $\varnothing 29$ DNA polymerase, and $1 \times$ Isothermal Reaction Buffer $(50 \mathrm{mM}$ Tris- $\mathrm{HCl}(\mathrm{pH} 7.5), 10 \mathrm{mM} \mathrm{MgCl} 2,200 \mu \mathrm{g} / \mathrm{ml}$ acetylated BSA) to a final volume of $25 \mu$. Of note, due to its high strand-displacing activity, Phage ø29 DNA polymerase was added last to the reaction mixture, which was then immediately incubated at $31^{\circ} \mathrm{C}$ for $1 \mathrm{~h}$ to perform RCA, followed by $70^{\circ} \mathrm{C}$ for $10 \mathrm{~min}$ to inactivate the polymerase and stop the reaction.

\section{Application to huntington CAG tandem repeats}

To apply our method for Huntington CAG tandem repeat, a 12nt Alexa 488 labeled PNA (5'-Alexa488CTGCTGCTGCTG, PNA Bio Inc.) was used to label the CAG trinucleotide repeat oligonucleotide. The CAG repeat oligonucleotide contains $66 \mathrm{CAG}$ trinucleotide repeats $\left((\mathrm{CAG})_{66}\right.$, Integrated DNA Technologies, Inc.), which allows the hybridization of a maximum of 16 PNA probes. The protocol for fluorescent PNA hybridization is similar to that in our PHAST method described above. $500 \mathrm{pM}$ CAG repeat oligonucleotides were mixed directly with 500 nM PNA in PNA hybridization buffer, incubated at $85^{\circ} \mathrm{C}$ for $5 \mathrm{~min}$ and left at room temperature in the dark for $2 \mathrm{~h}$ to complete the labeling. Then the purified sample was flowed through the microfluidic channel and detected on CICS.

\section{RESULTS}

\section{Overview of PHAST}

The PHAST protocol begins with total genomic DNA extracted from cells using standard phenol/chloroform extraction, taking care to avoid shearing the DNA molecules (Figure 1B). Telomeric DNA is pulled down using biotinylated PNA probes that hybridize to the telomere sequence, and streptavidin-coated magnetic beads. After the nontelomeric DNA has been discarded, the sample is heated to release the telomeres from the beads in the presence of excess Alexa488-labeled PNA (PNA-AF488). The magnetic beads are then discarded and the labeled telomeres are ready to be analyzed.

The sample is introduced into a microfluidic device on a custom-built microfluidic single molecule detection (SMD) instrument as described previously (Figure 1C) (30-32). Briefly, the microchannel spatially confines each passing PNA-AF488 labeled telomere into the path of a laser beam, in a manner analogous to the sheath/core flow configuration in flow cytometry. Typical SMD instruments utilize a diffraction-limited laser spot with a Gaussian profile to excite the fluorescently labeled targets (37). As a result, the brightness of each single molecule fluorescence burst depends on both the number of dyes on the molecule, as well as the excitation intensity through which it traverses. The inability to decouple these two contributing factors yields highly variable fluorescence signal from even a uniform sample, and limits SMD's ability to characterize individual molecules. To address these limitations, PHAST uses a cylindrical illumination confocal spectroscopy (CICS) configuration, wherein the excitation illumination is modified using beam-shaping optics to give an $O V$ in the shape of a uniform light sheet that extends across the entire microchannel cross-section (Figure 1C) (30-32). Not only does this allow quantification of the number of PNAAF488 probes on the telomeres, but it also ensures that every single telomere molecule passing through the microchannel is accounted for, thereby eliminating sampling bias, and in principle permitting the use of very few cells for quantification.

\section{Single-molecule fluorescence burst analysis of telomeres}

Events of single-molecule fluorescence bursts of telomeres are identified from the raw fluorescence signals using a predetermined threshold (Figure 2A). The time interval between the points at which the signal crosses a lower baseline level along the rising and falling edges is defined as the Width of the burst. The maximum intensity and total fluorescence (less the baseline) of each burst are the Height and Size, respectively. This thresholding procedure ensures that a fluorescent molecule, which barely exceeds the threshold, will not register as multiple events, were its signal to fluctuate around the threshold. While early studies involving CICS used the Size exclusively for analysis, recent development has extended its capabilities to other parameters such as the shape of the fluorescence bursts, in the form of packing density of DNA in buffers of different ionic strength (38), and the analysis of fluorescence distribution of entire sample populations (32).

In PHAST, the parameters (i.e. Size, Width and Height) are employed, together with a theoretical framework based on a model of peak parameters (Supplementary Note 2) to formulate a strategy for isolating true signal bursts from spurious ones. Depending on the background noise level, some spurious bursts will invariably be acquired due to signal fluctuation (Figure 2B). Such peaks can be efficiently removed by selecting a sufficiently high threshold at the expense of assay sensitivity. However, since the abundance of the shortest telomeres are of greatest interest, our chosen threshold, which corresponds to TLs of as little as $65 \mathrm{bp}$, is low enough to pick up a significant number of spurious peaks, particularly in the presence of relatively large quantities of residual non-telomeric DNA (Supplementary Note 4). These real and spurious peaks form two distinct clusters in a Width/Size plot (Figure 2C). This is because the back- 
A

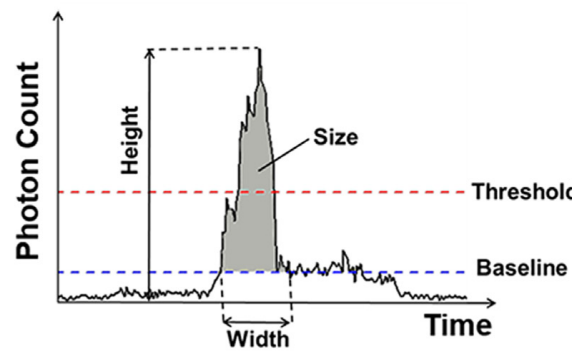

C

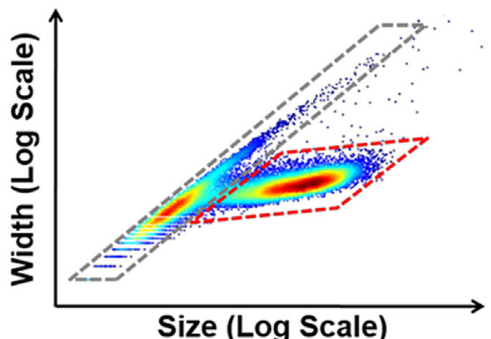

$E$

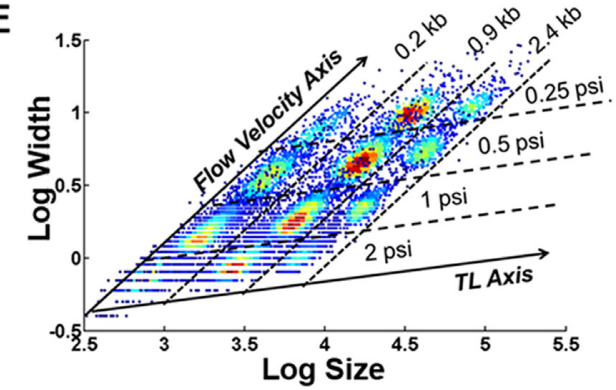

B

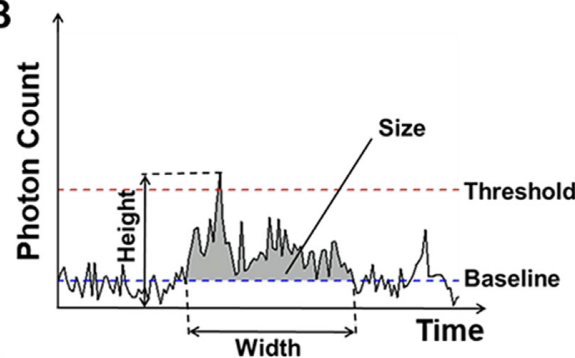

D
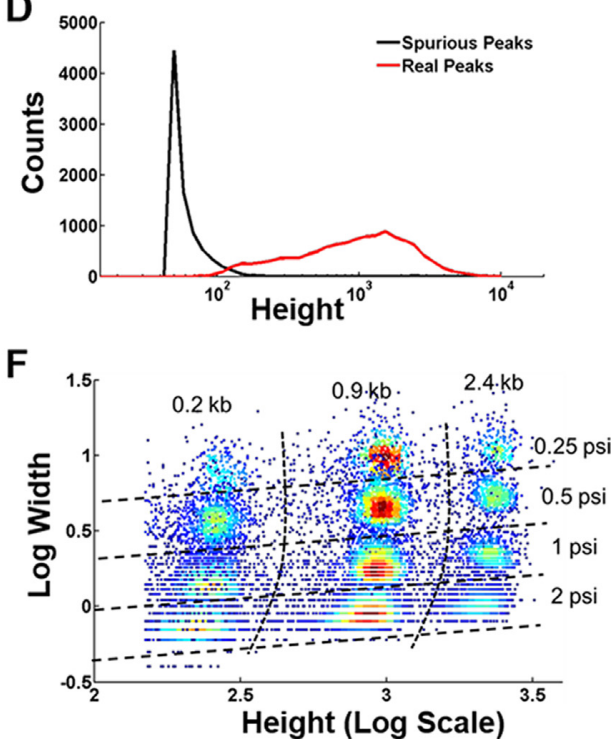

Figure 2. Analysis of PHAST data. (A) A fluorescent molecule is detected when a pre-determined threshold is exceeded. Each peak can be characterized by its Height, Width and total photon count, or Size, as illustrated. (B) Due to noise in the detector, the fluctuation in the background can occasionally exceed the threshold, whereupon a spurious peak, characterized by signal levels that hover around the baseline, and frequently large Width for any given Size, is detected. These spurious peaks can be removed by using a sufficiently high threshold, at the expense of assay sensitivity. (C) Alternatively, upon plotting on a $\log ($ Size $)-\log ($ Width $)$ plot, the peaks form two distinct clusters which can be separated by gating (Supplementary Note 5). (D) The real signal (red trace) can thus be distinguished from the spurious peaks (black trace), even when the shortest real peaks are shorter than the tallest spurious ones, as evidenced by the overlap between the two distributions. This cannot be achieved using a simple thresholding approach. (E) Although the Size parameter is, in principle, the most complete measure of the number of fluorophores on each telomere molecule, it is heavily dependent on the flow velocity. In fact, each Width-Size coordinate uniquely describes a telomere of a particular length, passing through the detection volume at a given velocity. (F) On the other hand, the Height parameter is very insensitive to flow velocity, with only a $10 \%$ decrease in the height when the velocity increases 8 -fold (driving pressure scales linearly with inverse velocity). As such, the peak height is selected as the measure of telomere length.

ground drifts that cause the spurious peaks typically hover around the baseline, with a nominal burst Size that is proportional to Width, i.e. the spurious cluster will have a slope of $\sim 1$ on a log-log plot of the two parameters. On the other hand, the physical constraints of the $O V$ size, DNA length, and flow velocity place limits on the possible Width values of peaks associated with the detection of actual fluorescent molecules, and as such the corresponding cluster will have a gentler slope on the same plot. This is corroborated by the theoretical estimates (slope $\sim 0.2$, Supplementary Note 2). Based on these distinguishing features, a gate is constructed that can isolate the spurious cluster (Figure 2C, D, Supplementary Note 5).

One limitation of previous implementations of CICS is that, since the total number of photons in a burst is proportional to the residence time in the laser excitation volume, comparison between different samples can only be made if the flow velocity is accounted for. Furthermore, because the pressure-velocity relationship depends on a number of factors, including microchannel geometry variation, surface properties, etc. the actual velocity in each experiment cannot be determined easily. By analyzing the parameters of telomere standards of different lengths $(0.2,0.9$ and $2.4 \mathrm{~kb})$ run through the PHAST instrument at different pressures, the effects of velocity on PHAST parameters are apparent (Figure 2E, F). Positions of the clusters in the Width/Size plot can be decoupled into two components, along the Flow Velocity Axis and Telomere Length Axis. Since both Width and Size scale linearly with flow velocity, the Flow Velocity Axis has a slope of $\sim 1$. This allows the same gate defined previously for removing spurious peaks to be used regardless of flow velocity. In fact, we found that the flow velocity had minimal effect on the burst Height, with only a $10 \%$ change when the flow velocity is increased 8-fold (Figure $2 \mathrm{~F})$. This translates to a smaller coefficient of variation (CV) for Height $(10 \%)$ compared with Size $(25 \%)$ in the length estimation of a $2.4 \mathrm{~kb}$ standard. Therefore Height is chosen as the parameter for telomere length estimation. Given the parallels between this CICS-based fluorescence spectroscopic approach and flow cytometry, we have named it flu- 
orescence flow moriometry (Gr. mório, molecule or small particle, + metron, to measure), of which PHAST is one of many applications.

\section{Measurement of telomere standards}

Based on the values derived from telomere standards of known lengths ( 0.1 to $2.4 \mathrm{~kb}$ ), burst Height is related to telomere length through a simple linear relationship (Figure $3 \mathrm{~A}$, Supplementary Note 3). The TL estimates has a CV of around $10-25 \%$, with greater uncertainty for shorter telomeres (Figure 3A, B), as well as very good reproducibility for three independent sets of standards tested at 6-month intervals $(\mathrm{CV}<11 \%$, Figure $3 \mathrm{~A}$, Table 1$)$. Although we expect the linear relationship to break down for much longer telomeres, it remains a valid approximation for up to 10-15 $\mathrm{kb}$ telomeres (the average TL of neonates) $(8,26)$, and underestimates the actual TL by only $15-20 \%$ for $30 \mathrm{~kb}$ telomeres, which is far longer than telomeres normally found in humans (Supplementary Note 3 ). To ensure that the gating procedure does not skew the TL estimates, a $2.4 \mathrm{~kb}$ telomere sample was tested with and without genomic DNA background. The resulting TL distribution was found to be unaffected by the gating procedure (Figure 3C).

\section{Determining proportion of short telomeres}

Although it has long been known that critically short telomeres can have an outsized impact on cell phenotype, what constitutes this critical threshold has, frustratingly, been poorly defined. This is primarily due to the absence of tools that can reliably size short telomeres. We have found only one study that determined, using STELA and sequencing, that $\mathrm{XpYp} / 17 \mathrm{p}$ fusion occurs below a minimal threshold of 13 hexanucleotide repeats, which is needed for telomeres to function normally (14). However, the method is not widely used, possibly due to the laborious and specific nature of the methodology. Nevertheless, the study confirms that a physical limit on functional TL exists, below which the protective functions of telomeres are lost, perhaps due to insufficient binding of telomeric repeat binding factors (e.g. $T R F 1, T R F 2$ ) (14). The only conventional measurement method that can routinely provide estimates for absolute TL is TRF. However, for the reasons stated in the introduction and elaborated in Supplementary Note 6, it is not ideal for determining the proportion of short telomeres.

To test the suitability of PHAST for this task, we prepared mixtures of short (200 bp) and relatively long (900 bp) telomeres at varying ratios. The short sub-population can be clearly distinguished in the histograms (Figure 3D). In addition, plotting the nominal fraction of $200 \mathrm{bp}$ standards in each sample with the measured fraction yielded a linear fit with a gradient very close to one (Figure 3E). The small offset in the observed fraction is attributed to incomplete labeling of a small proportion of the longer telomeres. It was reported that as few as five critically short telomeres out of 92 in a metaphase cell (i.e. $\sim 5 \%$ ) is sufficient to trigger the onset of senescence, highlighting the dominant effect of the shortest telomeres on cellular phenotype $(3,39)$. The ability of PHAST to differentiate between the proportion of very short telomeres in the $0 \%$ and $5 \%$ samples demonstrates its suitability for this purpose.

\section{Comparison between PHAST and q-FISH}

In general, PNA binding to the target DNA is not only very robust, but also highly sequence-specific, as evidenced by large changes in melting temperatures resulting from single nucleotide mismatches $(40,41)$. However, total genomic DNA contains a large excess of non-telomeric sequences. In a typical patient sample, telomeric DNA $(\sim 8 \mathrm{~kb}$ per chromosomal arm, or $0.75 \mathrm{Mb}$ per cell) constitutes only $\sim 0.01-0.02 \%$ of the total genomic DNA ( $\sim 6 \mathrm{~Gb})$. Consequently, we found that even the limited non-Watson-Crick interactions between PNA and DNA can yield an unacceptably high fluorescence background which can obscure short telomere peaks ( $<300 \mathrm{bp})(42)$.

PHAST utilizes a one-tube purification protocol to remove practically all non-telomeric DNA molecules, without biasing the TL distribution (Supplementary Figures S4, S5 and S10). Extracted DNA from cell lines (WI38, U2OS and R83) was purified using this method, and analyzed by PHAST. Because of the 100\% mass detection efficiency, there is minimal sampling bias and a reasonably good estimate of the TL distribution is obtained with as few as five cell equivalents ( $\sim 500$ peaks, Supplementary Figure S8). However, since the estimate improves with the number of sampled peaks, at least 1900 peaks are collected in each sample derived from cells, corresponding to 20 genomic equivalents (or 10 metaphases). The sizing results using PHAST is compared with those from q-FISH for up to 30 cells using quantile-quantile $(Q-Q)$ plots, which permits comparison of distributions of different units, with different number of elements (Figure 3F). For all three cell lines, the correlation between the PHAST and q-FISH results was largely linear, indicating that the measured telomere distributions were of the same shape for both methods. The slight deviation from linearity at the lower quantiles is attributed in part to artifacts of the q-FISH estimates (Supplementary Note 8). Clinical samples (one control, two from cancer patients), were also tested, and both PHAST and q-FISH found little difference between the samples (Supplementary Note 10). This is not unexpected, since the relationship between telomere length and cancer is complex.

\section{Shortening of telomeres with population doubling}

While commercial, immortalized cell lines including those in the previous section often possess some form of telomere lengthening or maintenance mechanism, other cell types, including primary cells, experience significant telomere shortening with each cell division, eventually leading to the onset of senescence. To verify our ability to detect such changes, excised human foreskin fibroblasts were cultivated for 38 population doublings (PD), and the cells were collected at various stages. PHAST was performed on the cells at PD $=$ $8,20,32$ and 38 , while q-FISH was performed at $\mathrm{PD}=8,14$, 20, 26 and 32 (Figure 4). At PD = 38, the cell division happens so infrequently that no metaphase could be obtained for q-FISH. Comparing the results for the two methods, we noticed that both showed shortening trends in the telomere length. Moreover, extrapolation of the PHAST results suggests that the initial telomere length $(P D=0)$ was approximately $8-9 \mathrm{~kb}$ (Figure $4 \mathrm{C}(\mathrm{i})$ ), which is a reasonable estimate corroborated by existing flow-FISH data (25). The 

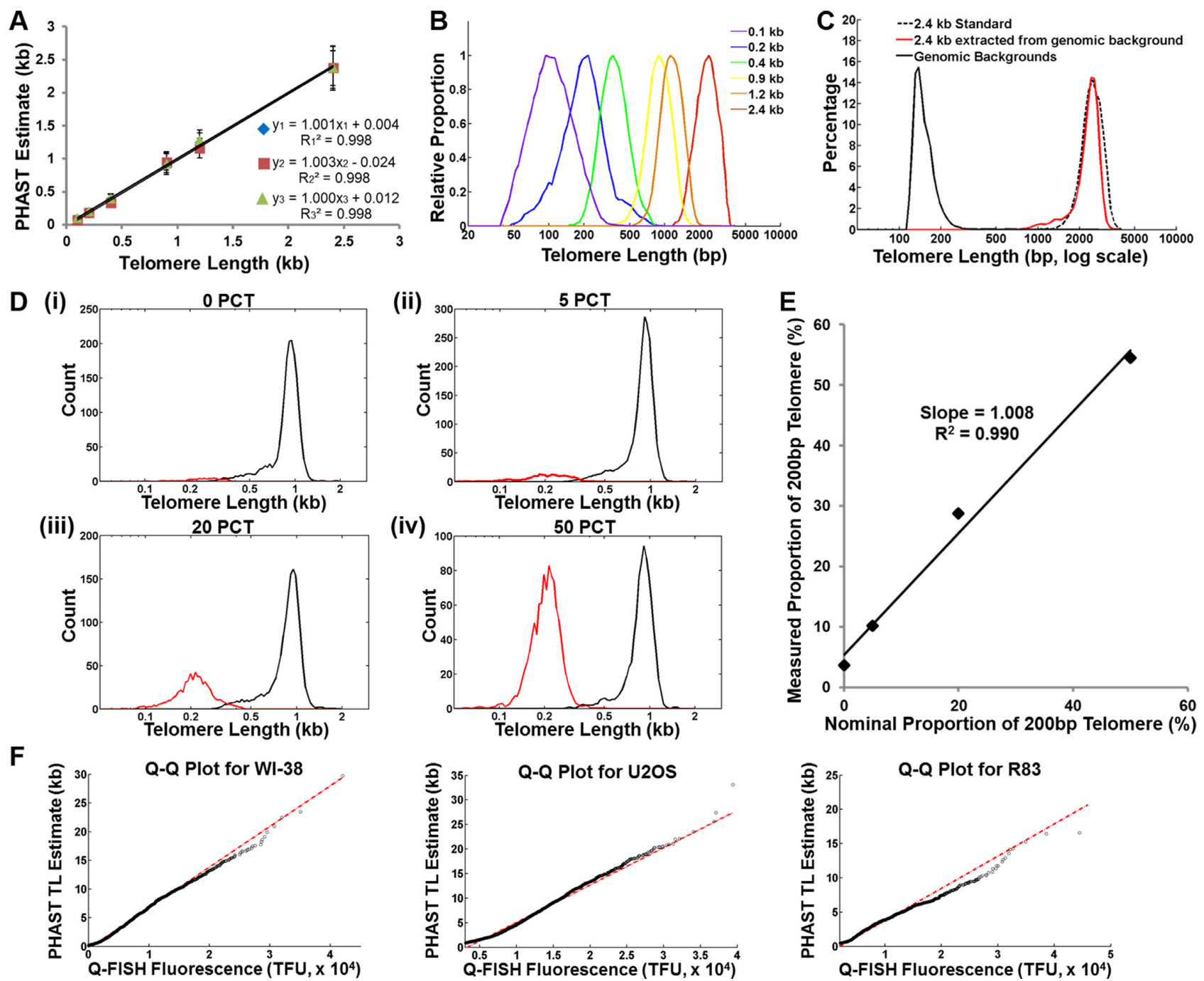

Figure 3. Telomere length (TL) determination using peak height. (A) Peak height is converted to TL using a simple linear equation derived from telomere standards of known lengths. The resulting three sets of TL estimates are nearly identical despite having been performed at 6-month intervals, with high linearity $\left(R^{2}>0.99\right)$. This reproducibility demonstrates the ability of PHAST to provide absolute telomere lengths with minimal calibration. (B) The six standards can be distinguished from each other by PHAST even when pre-mixed prior to detection, and have a smaller CV as the TL increases, as evidenced by the narrowing of the distributions with longer TL. (C) We have demonstrated the robustness of PHAST, by detecting a $2.4 \mathrm{~kb}$ telomere standard with and without a large excess of genomic DNA. Because the spurious peaks far outnumber the real peaks (by a factor of 10 to 1 ), the traces are normalized for clarity. After gating, the sample with genomic DNA (red solid trace) was essentially the same as that without any genomic DNA (black dashed trace). (D) To determine the ability to detect short telomeres using PHAST, 200-bp telomere standard is mixed at varying proportions with the 900-bp standard (0-50\%). (E) After gating and counting the two sub-populations, the measured proportion was plotted against the nominal proportion. The two numbers were very consistent, with a slightly higher proportion measured than expected. This is attributed to incomplete labeling of some of the longer telomeres, which are misidentified as short telomeres. (F) $Q-Q$ plots for the telomere estimates determined by PHAST and q-FISH are presented with three different cell lines (WI-83, U2OS and R83). This allows the distributions between two measurement methods to be compared directly even in the absence of common units and when the number of data points differs. Although PHAST and q-FISH are different methods, they yielded similar distribution shapes, as evidenced by the largely linear relationship between the corresponding quantiles. The red dashed line in each plot is the extrapolation of the interquartile range.

Table 1. CVs for telomere standards on CICS

\begin{tabular}{|c|c|c|c|c|c|}
\hline$\underline{\mathrm{TL}}(\mathrm{kb})$ & $\begin{array}{c}\text { TL Estimates Set } 1 \\
(\mathrm{~kb})\end{array}$ & $\begin{array}{c}\text { TL Estimates Set } 2 \\
(\mathrm{~kb})\end{array}$ & $\begin{array}{l}\text { TL Estimates Set } 3 \\
(\mathrm{~kb})\end{array}$ & $\begin{array}{l}\text { CV of TL Estimate } \\
\text { for Sets 1-3 }\end{array}$ & $\begin{array}{c}\text { TL Estimates } \\
\text { Simultaneous Detection } \\
(\mathrm{kb})\end{array}$ \\
\hline 0.1 & $0.09 \pm 0.01$ & $0.08 \pm 0.01$ & $0.10 \pm 0.01$ & $11.0 \%$ & $0.08 \pm 0.02$ \\
\hline 0.4 & $0.39 \pm 0.06$ & $0.34 \pm 0.05$ & $0.40 \pm 0.07$ & $9.0 \%$ & $0.41 \pm 0.05$ \\
\hline 0.9 & $0.97 \pm 0.12$ & $0.96 \pm 0.15$ & $0.92 \pm 0.15$ & $3.7 \%$ & $0.91 \pm 0.14$ \\
\hline 1.2 & $1.25 \pm 0.14$ & $1.17 \pm 0.14$ & $1.28 \pm 0.15$ & $4.4 \%$ & $1.29 \pm 0.16$ \\
\hline
\end{tabular}




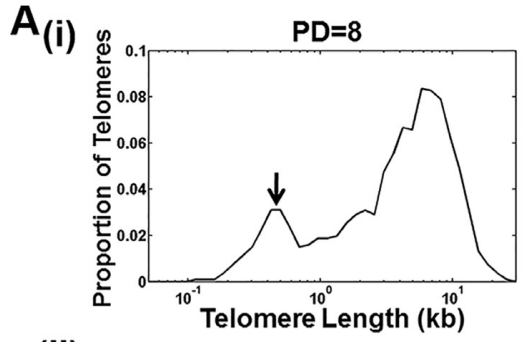

(ii)

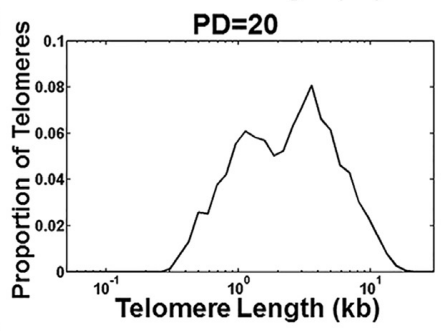

(iii)

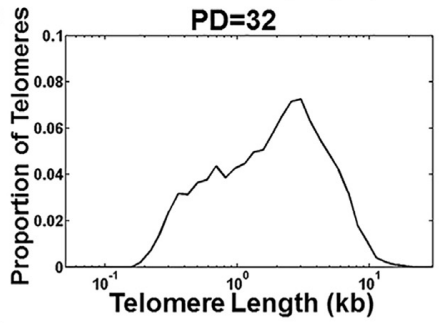

(iv)

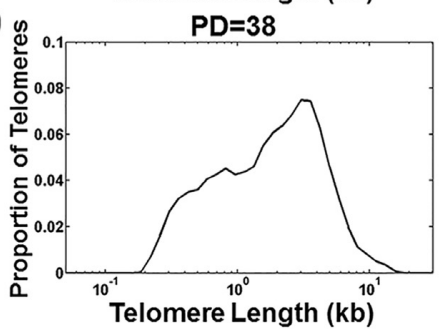

$B_{(i)}$

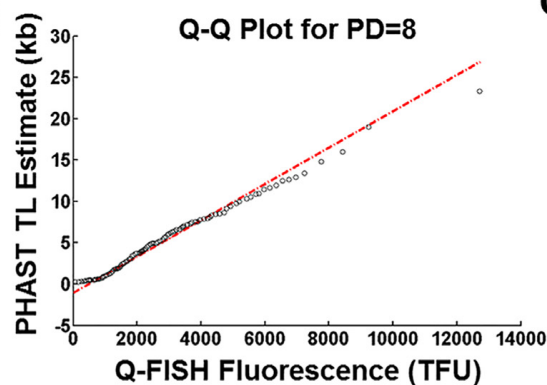

(ii)

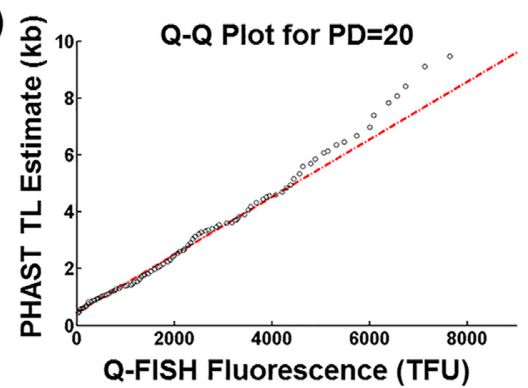

(iii)

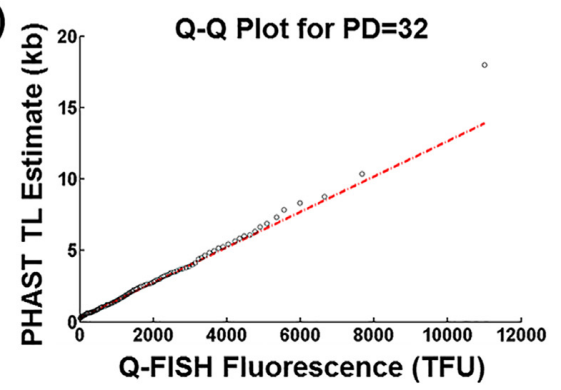

C

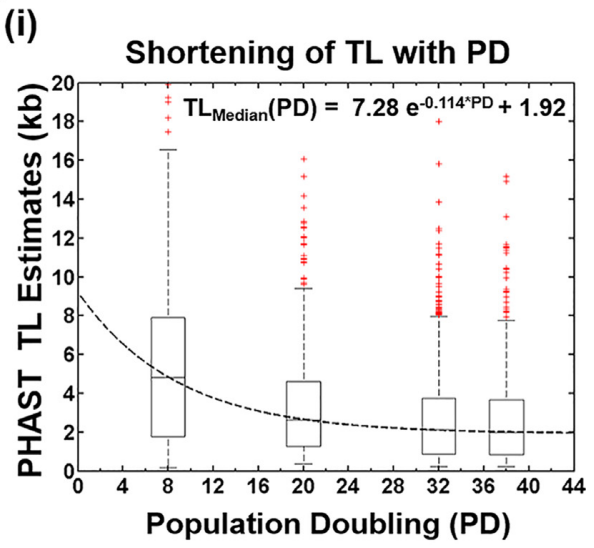

(ii)

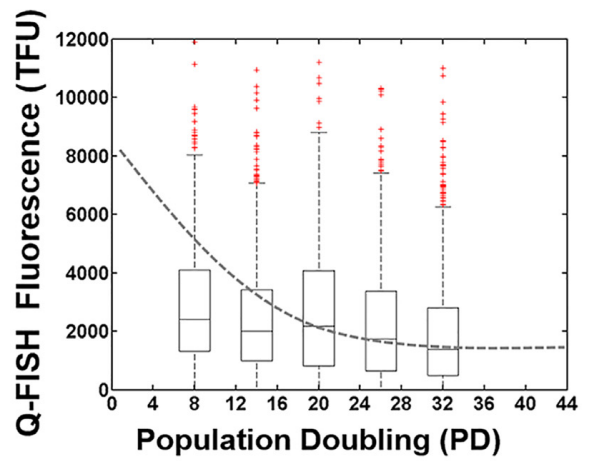

Figure 4. Detection of telomere shortening in primary cells with population doubling (PD). (A) Telomere shortening can be observed going from PD $=$ $8,20,32$, to 38 (i-iv). A sub-population of short telomeres was also observed at $\mathrm{PD}=8$. (B) The $Q-Q$ plots for PD $=8,20$ and 32 between PHAST and q-FISH. Since these samples were normalized with the same standards for q-FISH to obtain the TFU, one would expect all three to have the same slopes. However, the slope for $\mathrm{PD}=8\left(2.2 \times 10^{-3}\right)$ is around twice that of the other samples $\left(1.0 \times 10^{-3}\right.$ and $1.2 \times 10^{-3}$ for $\mathrm{PD}=20$ and $\mathrm{PD}=32$, respectively). This suggests that the TL determined by q-FISH is around half as long as expected from the PHAST results. (C) This difference is further confirmed by plotting the TL measurements against PD. (i) The median PHAST TL estimates (black dashed lines) were fitted to an exponential function. By setting the PD to 0 and $\infty$, we obtain TL estimates for the original patient cells and the cells at senescence, which are around $8-9 \mathrm{~kb}$ and $2 \mathrm{~kb}$, respectively. (ii) The q-FISH results showed some degree of shortening at late passages (20-32), but stayed relatively constant at early passages (PD 8 and 14). The dashed lines show the trend an exponential decay might be expected to follow. The shorter than expected TL at low PDs is most likely the result of an artifact of the method.

estimated median telomere length at $\mathrm{PD} \rightarrow \infty$ (i.e. at senescence) is around $2 \mathrm{~kb}$, which is close to that theorized by the leading experts in this field $(1,43)$. While these extrapolated estimates are not meant to be quantitative, the fact that they lie within the expected range further suggests that our telomere measurements are at least reasonably accurate.

One somewhat surprising observation was that at $\mathrm{PD}=$ 8 and 14, the telomere length determined using q-FISH was significantly shorter than expected, resulting in a kink in the trend. One possible explanation for this is heterogeneity of the cells in culture, where a small subpopulation of cells (potentially of a different type) divides rapidly early on and approaches replicative senescence, before a second, relatively slow-dividing subpopulation takes over. Because q-FISH analysis can only be applied to actively proliferating cells (since cells must be arrested in metaphase prior to probe hy- bridization) $(22,44)$, it may be detecting only the early subpopulation, for which the actual number of cell divisions may be far larger than the nominal PD values. On the other hand, because PHAST measures the telomere lengths of all the cells within a sample, it suffers from no such bias. This hypothesis is supported by the PHAST results, which show a secondary peak corresponding to short telomeres (Figure 4A(i), black arrow). As a result, the telomere length as determined by q-FISH may underestimate the telomere length for the whole population.

\section{DISCUSSION}

We have developed a powerful method to determine the distribution of telomere lengths within a population of cells. Apart from routine DNA extraction, the entire sample han- 
dling process for PHAST can be completed within 2-3 h. It addresses needs that are not met by two FISH-based methods that have emerged as leading candidates for telomere measurement, namely Flow-FISH and q-FISH.

Although Flow-FISH provides a useful clinical index for studying telomere-related disease states, the fact that it has to take into account the age of the subject suggests that the average TL information obtained is, at best, a proxy for the underlying biological trigger, most likely the accumulation of critically short telomeres. Therefore, it will fail to identify cases wherein the telomere distribution deviates from what is typical, either due to a broader distribution (Figure 1A(ii)), or catastrophic loss of telomeres (Figure 1A(iii)). The ability to test the TL in just non-adherent cells, rather than in relevant parenchymal cells (26), also limits its utility in understanding the role of telomeres in the development of many cancers.

On the other hand, q-FISH can determine not just TL distribution, but also chromosome-specific TL $(22,45)$. However, chromosome-specific telomere sizing may be extraneous since the biological effects of telomeres appear to be exerted primarily by the shortest telomeres, regardless of the chromosomal arms on which they are located (10). Furthermore, the requirement for live cells that can be induced into metaphase precludes using the method on archival samples or senescent cells. Even when applicable, qFISH is still an extremely laborious technique, taking hours to days to process a single sample, which often needs to be cultured and synchronized $(13,22)$. Lastly, q-FISH provides the telomere lengths in TFU, rather than actual telomere length, making comparison across experiments particularly difficult.

In contrast, the PHAST assay is capable of providing TL distribution for dividing or senescent cells; adherent or non-adherent cells; and frozen cell pellets or extracted DNA from sample banks. To our knowledge, PHAST is also unique in its ability to provide absolute telomere size distribution without needing concurrent measurement of standards, making it far more convenient. Because PHAST does not depend on any specific properties of the telomeric structures, instead taking a simple hybridization approach, it can be extended to other tandem repeats, many of which are implicated in severe neurological syndromes including Huntington's disease $(46,47)$ (Supplementary Note 11). Length variations in these sequences have also been implicated in the rapid morphological changes in the domesticated dog (48). While existing tandem repeat assays can be performed fairly routinely, they still suffer from technical and biological challenges including large allele dropout and null alleles that may be caused by primer site mutation $(49,50)$. As a result, tandem repeats remain an underexplored field, with the vast majority of studies focusing on short microsatellites $(51,52)$. In such instances, a PHASTbased approach can be advantageous in enabling detection without regard for the flanking sequences, and also eliminating biases arising from the amplification process. Furthermore, PHAST performs better for longer repeats $(e . g$. $>200 \mathrm{bp}$ ), and thus complements the existing approaches for analyzing tandem repeats.

In summary, we have demonstrated a new method that is able to determine the length of telomeres in extracted
DNA rather than whole cells. By utilizing a powerful single molecule detection approach, this allows us to determine the length of individual telomere molecules, the load of critically short telomeres, and the distribution of telomere lengths in a sample, rather than merely average telomere lengths. This confers to it many of the advantages of qFISH, but still able to work with extracted DNA and samples from frozen tissue banks.

\section{SUPPLEMENTARY DATA}

Supplementary Data are available at NAR Online.

\section{ACKNOWLEDGEMENTS}

The authors would like to thank Ying Wang (Georgetown University Medical Center) for technical assistance.

\section{FUNDING}

National Institutes of Health [R01CA155305, R21CA186809, R44GM103356, R01AI117032 to T.H.W. and R01CA132996 to Y.L.Z.]; Agency for Science, Technology and Research Singapore (A*STAR Scholarship to C.W.B.). Funding for open access charge: National Institutes of Health.

Conflict of interest statement. None declared.

\section{REFERENCES}

1. Levy,M.Z., Allsopp,R.C., Futcher,A.B., Greider,C.W. and Harley,C.B. (1992) Telomere end-replication problem and cell aging. J. Mol. Biol., 225, 951-960.

2. Sarek,G., Marzec,P., Margalef,P. and Boulton,S.J. (2015) Molecular basis of telomere dysfunction in human genetic diseases. Nat. Struct. Mol. Biol., 22, 867-874.

3. Armanios,M. and Blackburn,E.H. (2012) The telomere syndromes. Nat. Rev. Genet., 13, 693-704.

4. Oeseburg,H., de Boer,R.A., van Gilst,W.H. and van der Harst,P. (2010) Telomere biology in healthy aging and disease. Pflügers Arch.-Eur. J. Physiol., 459, 259-268.

5. Murnane,J.P. and Sabatier,L. (2004) Chromosome rearrangements resulting from telomere dysfunction and their role in cancer. Bioessays, 26, 1164-1174.

6. Thompson,S.L. and Compton,D.A. (2011) Chromosomes and cancer cells. Chromosome Res., 19, 433-444.

7. Xu,L., Li,S. and Stohr,B.A. (2013) The role of telomere biology in cancer. Annu. Rev. Pathol.: Mechan. Dis., 8, 49-78.

8. Lansdorp,P.M., Verwoerd,N.P., Van De Rijke,F.M., Dragowska,V., Little,M.-T., Dirks,R.W., Raap,A.K. and Tanke,H.J. (1996) Heterogeneity in telomere length of human chromosomes. Hum. Mol. Genet., 5, 685-691.

9. Martens,U.M., Zijlmans,J.M.J.M., Poon,S.S.S., Dragowska,W., Yui,J., Chavez,E.A., Ward,R.K. and Lansdorp,P.M. (1998) Short telomeres on human chromosome 17p. Nat. Genet., 18, 76-80.

10. Hemann,M., Strong,M., Hao,L. and Greider,C. (2001) The shortest telomere, not average telomere length, is critical for cell viability and chromosome stability. Cell, 107, 67-77.

11. Liu,Y., Kha,H., Ungrin,M., Robinson,M.O. and Harrington,L. (2002) Preferential maintenance of critically short telomeres in mammalian cells heterozygous for mTert. Proc. Natl. Acad. Sci. U.S.A., 99, 3597-3602.

12. Bendix,L., Horn,P.B., Jensen,U.B., Rubelj,I. and Kolvraa,S. (2010) The load of short telomeres, estimated by a new method, Universal STELA, correlates with number of senescent cells. Aging Cell, 9, 383-397.

13. Vera,E. and Blasco,M.A. (2012) Beyond average: potential for measurement of short telomeres. Aging (Albany NY), 4, 379. 
14. Capper,R., Britt-Compton,B., Tankimanova,M., Rowson,J., Letsolo,B., Man,S., Haughton,M. and Baird,D.M. (2007) The nature of telomere fusion and a definition of the critical telomere length in human cells. Genes Dev., 21, 2495-2508.

15. Abdallah,P., Luciano,P., Runge,K.W., Lisby,M., Géli,V., Gilson,E. and Teixeira,M.T. (2009) A two-step model for senescence triggered by a single critically short telomere. Nat. Cell Biol., 11, 988-993.

16. Gu,P., Min,J.N., Wang,Y., Huang,C., Peng,T., Chai,W. and Chang,S. (2012) CTC1 deletion results in defective telomere replication, leading to catastrophic telomere loss and stem cell exhaustion. EMBO J., 31, 2309-2321.

17. Lustig,A.J. (2003) Clues to catastrophic telomere loss in mammals from yeast telomere rapid deletion. Nat. Rev. Genet., 4, 916-923.

18. Bender,H.S., Murchison,E.P., Pickett,H.A., Deakin,J.E. Strong,M.A., Conlan,C., McMillan,D.A., Neumann,A.A., Greider,C.W. and Hannon,G.J. (2012) Extreme telomere length dimorphism in the tasmanian devil and related marsupials suggests parental control of telomere length. PLoS One, 7, e46195.

19. Ramunas,J., Yakubov,E., Brady,J.J., Corbel,S.Y., Holbrook,C., Brandt,M., Stein,J., Santiago,J.G., Cooke,J.P. and Blau,H.M. (2015) Transient delivery of modified mRNA encoding TERT rapidly extends telomeres in human cells. FASEB J., 29, 1930-1939.

20. de Jesus,B.B., Vera,E., Schneeberger,K., Tejera,A.M., Ayuso,E., Bosch,F. and Blasco,M.A. (2012) Telomerase gene therapy in adult and old mice delays aging and increases longevity without increasing cancer. EMBO Mol. Med., 4, 691-704.

21. Townsley,D.M., Dumitriu,B., Liu,D., Biancotto,A., Weinstein,B., Chen,C., Hardy,N., Mihalek,A.D., Lingala,S. and Kim,Y.J. (2016) Danazol treatment for telomere diseases. N. Engl. J. Med., 374, 1922-1931.

22. Aubert,G., Hills,M. and Lansdorp,P.M. (2012) Telomere length measurement - Caveats and a critical assessment of the available technologies and tools. Mut. Res./Fundam. Mol. Mech. Mutagen., 730, 59-67.

23. Walsh,K.M., Codd,V., Smirnov,I.V., Rice,T., Decker,P.A., Hansen,H.M., Kollmeyer,T., Kosel,M.L., Molinaro,A.M., McCoy,L.S. et al. (2014) Variants near TERT and TERC influencing telomere length are associated with high-grade glioma risk. Nat. Genet., 46, 731-735.

24. Codd,V., Nelson,C.P., Albrecht,E., Mangino,M., Deelen,J., Buxton,J.L., Hottenga,J.J., Fischer,K., Esko,T., Surakka,I. et al. (2013) Identification of seven loci affecting mean telomere length and their association with disease. Nat. Genet., 45, 422-427.

25. Aubert,G. and Lansdorp,P.M. (2008) Telomeres and aging. Physiol. Rev., 88, 557-579.

26. Aubert,G., Baerlocher,G.M., Vulto,I., Poon,S.S. and Lansdorp,P.M. (2012) Collapse of telomere homeostasis in hematopoietic cells caused by heterozygous mutations in telomerase genes. PLoS Genet., 8, e1002696.

27. Kimura,M., Stone,R.C., Hunt,S.C., Skurnick,J., Lu,X., Cao,X., Harley,C.B. and Aviv,A. (2010) Measurement of telomere length by the Southern blot analysis of terminal restriction fragment lengths. Nat. Protoc., 5, 1596-1607.

28. Oexle,K. (1998) Telomere length distribution and Southern blot analysis. J. Theor. Biol., 190, 369-377.

29. Baird,D.M., Rowson,J., Wynford-Thomas,D. and Kipling,D. (2003) Extensive allelic variation and ultrashort telomeres in senescent human cells. Nat. Genet., 33, 203-207.

30. Liu,K.J., Brock,M.V., Shih,I. and Wang,T.H. (2010) Decoding circulating nucleic acids in human serum using microfluidic single molecule spectroscopy. J. Am. Chem. Soc., 132, 5793-5798.

31. Liu,K.J. and Wang,T.-H. (2008) Cylindrical illumination confocal spectroscopy: rectifying the limitations of confocal single molecule spectroscopy through one-dimensional beam shaping. Biophys. J., 95 2964-2975.
32. Beh,C.W., Pan,D., Lee,J., Jiang,X., Liu,K.J., Mao,H.-Q. and Wang,T.-H. (2014) Direct interrogation of DNA content distribution in nanoparticles by a novel microfluidics-based single-particle analysis. Nano Lett., 14, 4729-4735.

33. Zheng,Y.L., Loffredo,C.A., Alberg,A.J., Yu,Z.P., Jones, R.T. Perlmutter,D., Enewold,L., Krasna,M.J., Yung,R., Shields,P.G. et al. (2005) Less efficient G(2)-M checkpoint is associated with an increased risk of lung cancer in African Americans. Cancer Res., 65, 9566-9573.

34. Zheng,Y.L., Zhou,X., Loffredo,C.A., Shields,P.G. and Sun,B. (2011) Telomere deficiencies on chromosomes $9 \mathrm{p}, 15 \mathrm{p}, 15 \mathrm{q}$ and $\mathrm{Xp}$ : potentia biomarkers for breast cancer risk. Hum. Mol. Genet., 20, 378-386.

35. Liu,D.Y., Daubendiek,S.L., Zillman,M.A., Ryan,K. and Kool,E.T. (1996) Rolling circle DNA synthesis: small circular oligonucleotides as efficient templates for DNA polymerases. J. Am. Chem. Soc., 118 1587-1594.

36. Lizardi,P.M., Huang,X.H., Zhu,Z.R., Bray-Ward,P., Thomas,D.C. and Ward,D.C. (1998) Mutation detection and single-molecule counting using isothermal rolling-circle amplification. Nat. Genet., 19, 225-232.

37. Zander,C., Keller,R.R. and Enderlein,J. (2002) Single Molecule Detection in Solution. Wiley Online Library.

38. Friedrich,S.M., Liu,K.J. and Wang,T.-H. (2015) Single molecule hydrodynamic separation allows sensitive and quantitative analysis of DNA conformation and binding interactions in free solution. J. Am. Chem. Soc., 138, 319-327.

39. Kaul,Z., Cesare,A.J., Huschtscha,L.I., Neumann,A.A. and Reddel,R.R. (2012) Five dysfunctional telomeres predict onset of senescence in human cells. EMBO Rep., 13, 52-59.

40. Ray,A. and Nordén,B. (2000) Peptide nucleic acid (PNA): its medical and biotechnical applications and promise for the future. FASEB J., 14, 1041-1060.

41. Park,H., Germini,A., Sforza,S., Corradini,R., Marchelli,R. and Knoll,W. (2007) Effect of ionic strength on PNA-DNA hybridization on surfaces and in solution. Biointerphases, 2, 80-88.

42. Tackett,A.J., Corey,D.R. and Raney,K.D. (2002) Non-Watson-Crick interactions between PNA and DNA inhibit the ATPase activity of bacteriophage T4 Dda helicase. Nucleic Acids Res., 30, 950-957.

43. Allsopp,R.C. and Harley,C.B. (1995) Evidence for a critical telomere length in senescent human fibroblasts. Exp. Cell Res., 219, 130-136.

44. Lauzon,W., Sanchez Dardon,J., Cameron,D.W. and Badley,A.D. (2000) Flow cytometric measurement of telomere length. Cytometry, 42, 159-164.

45. Zheng,Y.-L., Ambrosone,C., Byrne,C., Davis,W., Nesline,M. and McCann,S. (2010) Telomere length in blood cells and breast cancer risk: investigations in two case-control studies. Breast Cancer Res. Treat., 120, 769-775.

46. Gelfand,Y., Rodriguez,A. and Benson,G. (2007) TRDB - the tandem repeats database. Nucleic Acids Res., 35, D80-D87.

47. Margolis,R.L. and Ross,C.A. (2003) Diagnosis of Huntington disease. Clin. Chem., 49, 1726-1732.

48. Fondon,J.W. and Garner,H.R. (2004) Molecular origins of rapid and continuous morphological evolution. Proc. Natl. Acad. Sci. U.S.A., 101, 18058-18063.

49. Dewoody,J., Nason,J.D. and Hipkins, V.D. (2006) Mitigating scoring errors in microsatellite data from wild populations. Mol. Ecol. Notes, 6, 951-957.

50. Zane,L., Bargelloni,L. and Patarnello,T. (2002) Strategies for microsatellite isolation: a review. Mol. Ecol., 11, 1-16.

51. McIver,L., McCormick,J., Martin,A., Fondon,J. and Garner,H. (2013) Population-scale analysis of human microsatellites reveals novel sources of exonic variation. Gene, 516, 328-334.

52. Duitama,J., Zablotskaya,A., Gemayel,R., Jansen,A., Belet,S., Vermeesch,J.R., Verstrepen,K.J. and Froyen,G. (2014) Large-scale analysis of tandem repeat variability in the human genome. Nucleic Acids Res., 42, 5728-5741. 